\title{
Compressive sensing optical coherence tomography using randomly accessible lasers
}

\author{
Mark Harfouche $^{a}$, Naresh Satyan ${ }^{b, a}$, Arseny Vasilyev $^{a}$, and Amnon Yariv ${ }^{a}$ \\ ${ }^{a}$ California Institute of Technology, 1200 E. California Blvd. Pasadena, California, USA; \\ ${ }^{b}$ Telaris Inc., Santa Monica, California, USA
}

\begin{abstract}
We propose and demonstrate a novel a compressive sensing swept source optical coherence tomography (SSOCT) system that enables high speed images to be taken while maintaining the high resolution offered from a large bandwidth sweep.

Conventional SSOCT systems sweep the optical frequency of a laser $\omega(t)$ to determine the depth of the reflectors at a given lateral location. A scatterer located at delay $\tau$ appears as a sinusoid $\cos (\omega(t) \tau)$ at the photodetector. The finite optical chirp rate and the speed of analog to digital and digital to analog converters limit the acquisition rate of an axial scan. The proposed acquisition modality enables much faster image acquisition rates by interrogating the beat signal at randomly selected optical frequencies while preserving resolution and depth of field.

The system utilizes a randomly accessible laser, a modulated grating Y-branch laser, to sample the interference pattern from a scene at randomly selected optical frequencies over an optical bandwidth of $5 \mathrm{THz}$, corresponding to a resolution of $30 \mu \mathrm{m}$ in air. The depth profile is then reconstructed using an $l_{1}$ minimization algorithm with a LASSO constraint. Signal-dependent noise sources, shot noise and phase noise, are analyzed and taken into consideration during the recovery. Redundant dictionaries are used to improve the reconstruction of the depth profile. A compression by a factor of 10 for sparse targets up to a depth of $15 \mathrm{~mm}$ in noisy environments is shown.
\end{abstract}

Keywords: optical coherence tomography, random access laser, compressive sensing, LASSO, swept source

\section{INTRODUCTION}

Optical coherence tomography, (OCT), is becoming an active area of research for 3D biomedical imaging. ${ }^{1}$ OCT enables 3D depth profiles to be formed by taking a depth scan at a particular lateral location and moving the probing optical beam around the desired field of view to obtain the full 3D profile. Recent advancements in swept laser sources, such as MEMS VCSELS ${ }^{2}$ and Vernier tunable lasers ${ }^{3-5}$ have made it possible to measure the depth profile at a particular lateral location at rates of $100 \mathrm{~s} \mathrm{kHz}$. This scan rate results in signals of interest in the range of $100 \mathrm{~s} \mathrm{MHz}$ and limitations imposed by the finite speed of acquisition electronics prevent further increases in the scan rate. Physical constraints, such as the mechanical resonance of MEMS VCSELs, and carrier dynamics within the laser, currently pushed to their limits, ultimately limit the tuning speed of the optical source.

Here, we propose and demonstrate the use of compressive sampling ${ }^{6}$ in conjunction with a Vernier tunable laser to show that compressive sensing can be used to increase the acquisition rate of a depth scan at a particular lateral location. Vernier tunable lasers, such as the modulated grating Y-branch laser (MG-Y) used in this demonstration, are well suited for compressive acquisition schemes since they are not constrained to sequentially sweeping the optical frequency. In addition, they have been shown to be able to change the output optical frequency on a nanosecond timescale. ${ }^{7}$ In the following sections, we will review swept source optical coherence tomography, briefly describe the operation and dynamic characterization of Vernier tunable lasers, and demonstrate their use in a compressive sampling OCT setup.

Send correspondence to Mark Harfouche: mark.harfouche@caltech.edu.

Compressive Sensing III, edited by Fauzia Ahmad, Proc. of SPIE Vol. 9109,

91090L · C 2014 SPIE · CCC code: 0277-786X/14/\$18 · doi: 10.1117/12.2048754

Proc. of SPIE Vol. 9109 91090L-1 


\section{3D IMAGING USING SWEPT SOURCE OPTICAL COHERENCE TOMOGRAPHY}

We begin by reviewing the principles behind 3D imaging using a swept laser. While the principle behind swept source OCT (SSOCT) relies on changing the optical frequency $\omega$ of the laser with time, the rate of change of the optical frequency is slow enough that a quasi static analysis applies. In what follows, we consider the output at a given frequency and then allow the frequency to change with time.

To measure depth, SSOCT characterizes the interference between the reflected optical beam from the scatterers in the scene and that of a mirror at a known location. One configuration that enables this measurement is a Michelson interferometer shown on the right of Figure 1. The output of the laser is collimated and split into two paths by a beam splitter: half the light is directed towards the scene and the other half towards a reference mirror. The strong reflection from the mirror passes back through the beam splitter towards the photodetector and beats with scattered light from the scene. Light reflecting from an object located at a relative distance $d_{i}$ from the mirror, will be delayed by $\tau_{i}=2 n d_{i} / c$ where $n$ is the refractive index of the medium, and $c$ is the speed of light in vacuum. The reflections from the scatterers in the scene beat with the reference mirror and the measured voltage at the photodetector can be written as:

$$
V\left(\omega=\omega_{0}\right)=\frac{\rho P}{4}\left(1+\sum_{i} R_{i}+\sum_{i} 2 \sqrt{R_{i}} \cos \left(\omega_{0} \tau_{i}\right)\right)
$$

where $P$ is the output power from the laser, $\rho$ is the responsivity of the detector in $\mathrm{V} / \mathrm{W}$, and $R_{i}$ is the reflectivity of the $i^{\text {th }}$ reflector. Note that we have assumed $R_{i} \ll 1$ allowing the omission the beating terms involving $\sqrt{R_{i} R_{j}}, i \neq j$. For simplicity, we will set $\rho P / 4=1$ and ignore the $\sum_{i} R_{i}$ terms in subsequent discussions.

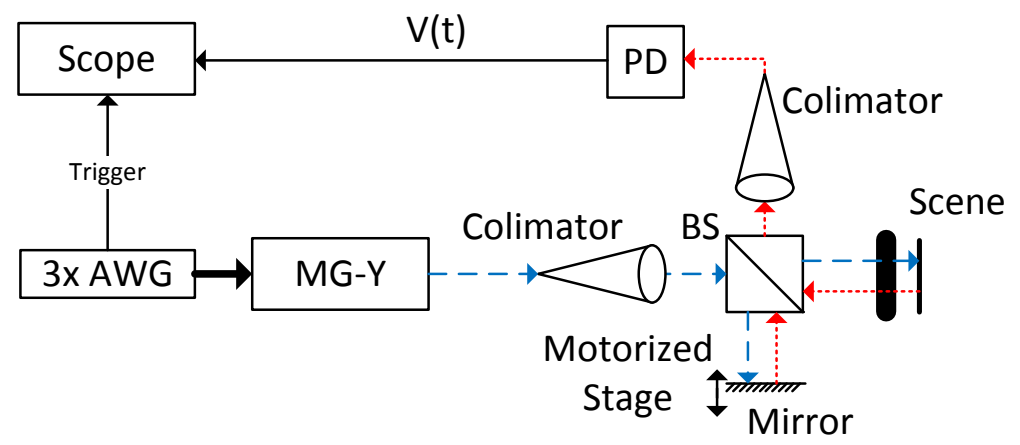

Figure 1: System schematic. The electrical path is shown in black. The output optical beam is shown in a dashed blue line while the useful optical return path is shown in a dotted red line. BS denotes a 50:50 beam splitter. The motorized stage enables the scene, made of acrylic slabs, to be measured at various levels of path length mismatch. MG-Y refers to the modulated grating Y-branch laser. $3 \times$ AWG refers to the three arbitrary waveform generators used to tune the laser. PD refers to the photodetector (Thorlabs PDA10CF).

In current SSOCT embodiments, $\omega$ is continuously varied by either changing the cavity length of the laser, such as in MEMS VCSELS, ${ }^{2}$ by tuning an optical filter, ${ }^{8}$ or by injecting current into the laser. ${ }^{9}$ Equation 1 can be written as:

$$
V\left(\omega=\omega_{0}+\Delta \omega(t)\right)=1+\sum_{i} 2 \sqrt{R_{i}} \cos \left(\omega_{0} \tau_{i}+\Delta \omega(t) \tau_{i}\right) \quad t \in[0, T) .
$$

The strength of the reflector at a given location can be reconstructed by taking the Fourier transform of $V(\Delta \omega(t))$. The conjugate Fourier variable to $\Delta \omega$ is the location of a reflector and the magnitude of the Fourier transform at that location is the reflectivity. The resolution of the system, $\delta d=c / 2 B$, is given by the optical sweep bandwidth, $B=\Delta \omega_{\max }-\Delta \omega_{\min }$, while the maximum measurable delay is determined by the coherence length of the laser. In current OCT embodiments, the function $\Delta \omega(t)$ is some known monotonically increasing or decreasing function in time. A full 3D measurement is obtained by scanning the output beam across the desired lateral field of view. ${ }^{1}$ 
Discretizing Equation 2 in the optical frequency $(\Delta \omega)$ and time delay domains $(\tau)$, we obtain:

$$
\mathbf{y}=\mathbf{1}+\mathbf{F x},
$$

where $\mathbf{y} \in \mathcal{R}^{N}$ contains the measurements $y_{j}=V\left(\Delta \omega\left(t_{j}\right)\right)$ with $t_{j}<t_{j+1}$, $\mathbf{x}$ is a vector of size $N$ contains the reflectivity of the scene at each Fourier limited resolution bin corresponding to a location $\tau_{i}$. $\mathbf{F}$ is an $N \times N$ is the Fourier matrix that relates the reflectivity at a given location to the measured beat signal at the photodetector.

The speed at which one can measure the full vector $\mathbf{y}$ is limited by the speed at which $\Delta \omega$ can be changed and by acquisition electronics. MEMS VCSELs are limited by their mechanical resonance, ${ }^{2}$ while FDML lasers ${ }^{8}$ are limited by the rotation speed of the tuning grating. Compressive sensing (CS) theory ${ }^{6}$ states that if the vector $\mathbf{x}$ is sparse, it can be reconstructed by taking a randomly chosen subset of the measurements $\mathbf{y}$ related to $\mathbf{x}$ by a partial Fourier matrix. The square measurement matrix $\mathbf{F}$ in Equation 3 is then replaced by matrix A with $m \ll N$ rows and $N$ columns made of randomly selected rows of the original $\mathbf{F}$ matrix. In SSOCT, this translates to measuring the beat signal at a subset of the optical frequencies. Unfortunately, typical laser sources used in SSOCT must be continuously tuned making them unsuitable for this type of acquisition. In the next sections, we will explore how Vernier tunable lasers can overcome this limitation. This class of lasers allows for arbitrary addressing of optical frequencies effectively reordering the rows of the Fourier measurement matrix F. If the rows are randomly ordered, any consecutive $m$ measurements $y_{1} \ldots y_{m}$ become sufficient to recover the depth profile $\mathbf{x}$ without sacrificing resolution or depth of field. The vector $\mathbf{x}$ containing the reflectivity of the scene can be recovered from $\mathbf{y}$ through a variety of algorithms ${ }^{6,10,11}$ thus decreasing the overall acquisition time for a depth scan. Prior to any CS reconstruction, the mean of the $m$ entries in the raw measurement is removed to form $\mathbf{y}$ to account for the strong DC term (1 in Equation 3).

\section{SAMPLED GRATING LASERS FOR COMPRESSED SENSING SSOCT}

Recently, a new class of single mode laser based on sampled grating resonator has been proposed for SSOCT. ${ }^{3,4,12}$ The principle behind these sampled grating lasers is that an arbitrary optical frequency can be selected by addressing a different mode of the resonator. The lasers achieve this by having two sampled grating reflectors with slightly different periods forming the optical cavity. Each grating is resonant at multiple frequencies, but the combined effect of the two grating is to allow oscillation at just a single optical frequency. The optical frequency is addressed by changing the refractive index of the individual resonators through local heating ${ }^{4}$ or carrier injection. ${ }^{13}$ This causes an optical mode-hop and can change the output of the laser by as much as $40 \mathrm{~nm}$. Unlike continuously tunable lasers, these lasers, known as Vernier tunable lasers, can address optical frequencies in any desired order making them versatile tools for compressive sensing.

In this study, we use a modulated grating Y-branch laser (Syntune 7500) as the randomly accessible tunable laser source in the $1550 \mathrm{~nm}$ range. The characterization of the source was accomplished by changing the tuning currents to the specified values using arbitrary waveform generators (AD9910) and waiting until all transients settle before measuring the optical frequency with an wavemeter (Burleigh WA-1100). Figure 2 shows the measured optical frequency of the laser as a function of two tuning currents, a third tuning current (not shown in the plot) is used to fine tune the output frequency.

The laser is tuned to 1000 different optical frequencies in a $1 \mathrm{~ms}$ interval. The nominal optical frequencies are chosen from $190 \mathrm{THz}$ to $195 \mathrm{THz}$ in $5 \mathrm{GHz}$ increments corresponding to a Nyquist limited depth of field of $15 \mathrm{~mm}$ with an axial resolution of $30 \mu \mathrm{m}$ in free space. The order of optical frequencies is randomized so as to allow any group of consecutive $m<N$ measurements to be sufficient to reconstruct the reflectivity profile of the scene. The CS reconstruction is performed by solving an $l_{1}$ minimization problem detailed in Section 6 .

\section{DYNAMIC CHARACTERIZATION OF THE MG-Y LASER}

One of the major problems in implementation of CS is that the measurement matrix $(\mathbf{A})$ is not always known with full precision. While this might manifest itself as blurring or lowered resolution in conventional measurement systems, knowledge of $\mathbf{A}$ will determine whether or not the signal is sparse in the chosen representation. If the sparsity in the chosen basis is reduced, then the number of measurements required to recover the original signal will also increase thus reducing the usefulness of compressive sampling. 


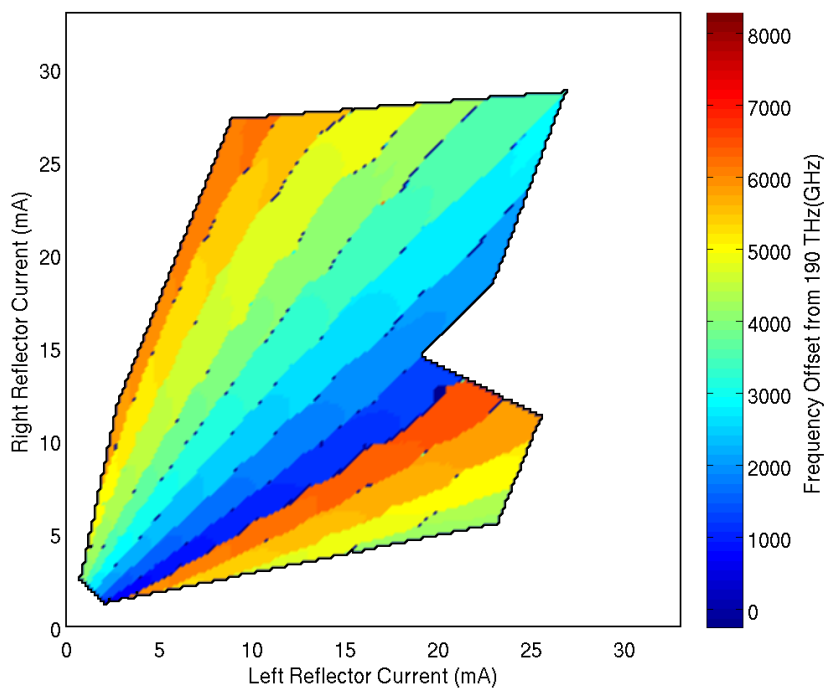

Figure 2: Color plot showing the addressable optical frequencies using the MG-Y laser by tuning the grating currents. Tuning from $190 \mathrm{THz}$ to $195 \mathrm{THz}$ is achievable giving the overall system an axial resolution of $30 \mu \mathrm{m}$ in free space. The regions shown in white were found to have an unstable optical frequency output and thus are not considered to be useful.

In the case of CS SSOCT, the exact output frequency of the laser needs to be known with high precision. Slow thermal transients heat the laser and appear as a systematic discrepancy between frequency characterized with the slow wavemeter and the actual output frequency of the MG-Y laser during a single sweep. To measure the error in the output optical frequency, we replace the scene by a strong mirror. If the measurements are reordered in ascending optical frequency, the error can be modeled as a phase modulation of the beat signal:

$$
V\left(\Delta \omega_{j}\right)=\cos \left(\omega_{0} \tau+\Delta \omega_{j} \tau+\delta_{j} \tau\right)
$$

where $\tau$ is the delay between the two mirrors, and $\delta_{j}$ is the deviation in the output optical frequency from $\omega_{0}+\Delta \omega_{j}$. For a nominally path length matched reflector, $\delta_{j} \tau$ is small and the error will not be visible in the measurement. For a larger delay, $\delta_{j} \tau$ becomes more significant and will appear as sidebands in the frequency domain. This is illustrated in Figure 3(a) where the CS reconstruction of the depth profile, detailed in Section 6, is performed with $m=200$ measurements for a reflector with a delay of $\sim 1 \mathrm{~mm}$ and an other with $\sim 9 \mathrm{~mm}$. While the target at a location of $1 \mathrm{~mm}$ appears as a single reflector, the target located at $\sim 9 \mathrm{~mm}$ appears as 4 reflectors with the magnitude of the strongest peak is reduced compared to the target at located $1 \mathrm{~mm}$.

To estimate $\delta_{j}$, a calibration step is performed prior to the measurement of the desired scene. The calibration process involves placing a strong mirror in the scene and measuring the signal at path length mismatches of $5,6,7$ and $8 \mathrm{~mm}$ by moving the reference mirror on the motorized stage. All 1000 optical frequency samples are reordered and the phase of the signal $\left(\delta_{j} \tau\right)$ is estimated using a Hilbert transform and assuming the true location of the mirror is at the peak of the Fourier transform. The average of $\delta_{j}$ is used as the true deviation. To show the effectiveness of this method, the CS reconstruction, as detailed in Section 6, of the single reflector scene is performed with $\Delta \omega_{j}$ actual $=\Delta \omega_{j}$ desired $+\delta_{j}$ and the results are shown in Figure 3(b). The false targets are removed restoring the sparsity of the signal and the strength of the true reflector.

\section{NOISE SOURCES IN CS SSOCT}

The two dominant sources in OCT are shot noise and phase noise. Unlike the canonical compressive sensing problem where the noise is independent of the vector $\mathbf{x}$, noise in OCT is dependent on the received optical power.

We start by defining variables for the noise in the signal space and the measurement space:

$$
\mathbf{y}=\mathbf{A}\left(\mathbf{x}_{\text {noiseless }}+\mathbf{n}\right)=\mathbf{A} \mathbf{x}_{\text {noiseless }}+\mathbf{A n}=\mathbf{y}_{\text {noiseless }}+\mathbf{z},
$$



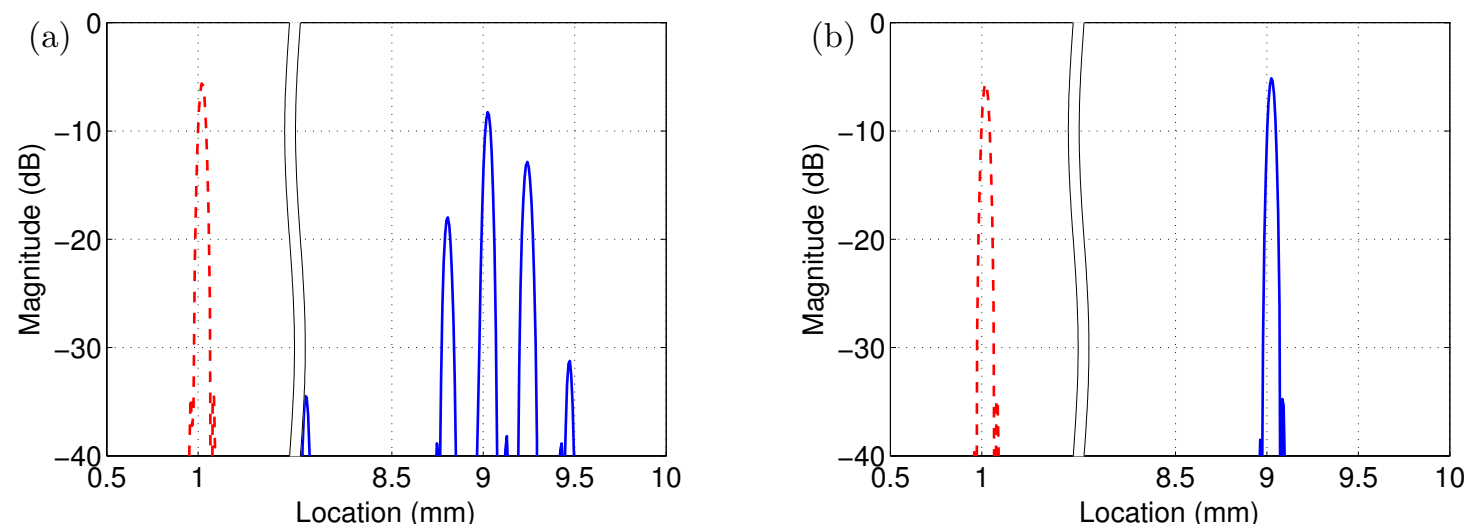

Figure 3: CS reconstruction of a scene with a single strong reflector. The dashed red line shows a strong reflector close to being path length matched. The solid blue line shows a strong reflector further away from the path length matching condition. The two different lines are obtained from independent measurements and reconstructed independently. (a) CS reconstructed of an uncalibrated measurement. The target at a small path length mismatch is properly reconstructed while the target further away has a smaller amplitude and appears as multiple targets. (b) CS reconstruction after recalibration of the measurement matrix. Both targets are properly reconstructed once the impairments in the measurement matrix have been calibrated.

where $\mathbf{n}$ is the noise in the signal itself due to shot noise and the phase noise of the laser, and $\mathbf{z}$ is the noise in the measurement domain. To simplify the discussion, we consider the case where the columns of $\mathbf{A}$ have unit norm, this can easily be achieved by rescaling the measurements. We will use the fact the approximation $\|x\|_{2}^{2} \approx\|y\|_{2}^{2}$, guaranteed by the restricted isometry property. ${ }^{14}$ It can also be shown that the expectation of the energy in the noise is also conserved giving rise to $\left\langle\|\mathbf{n}\|_{2}^{2}\right\rangle \approx\left\langle\|\mathbf{z}\|_{2}^{2}\right\rangle$ because the entries in $\mathbf{n}$ are assumed to be independent, $\langle\cdot\rangle$ denotes the expectation value.

Shot noise, originating from the arrival times of photons at the detector, is dependent on the total received power of the laser. In practice, two factors limit the output power of the source: the maximum achievable power from the laser, and the total energy the sample is able to withstand before being damaged. Shot noise therefore sets a upper bound on the signal to noise ration (SNR) of every measured sampled of $\sqrt{N_{e}}$ where $N_{e}$ is the number of photoelectrons collected by the detector. Since we are mostly interested in scenes consisting of weak reflectors, $N_{e}$ can be well approximated by the number of photons collected by the reference arm.

The expected power in the signal due to the phase noise, originating from the finite coherence time of the laser, is proportional to: ${ }^{15}$

$$
\left\langle\left\|\mathbf{n}_{\text {phase }}\right\|_{2}^{2}\right\rangle \propto \sum_{i} R_{i} \tau_{i}^{2}
$$

where $R_{i}$ is the reflectivity of the reflector, $\tau_{i}$ is the delay of the $i^{\text {th }}$ reflector. Two key observations should be made about the expression above. First, the total noise power is dependent on the strength of the reflectors $R_{i}$. Second, the amount of noise contributed by each reflector is dependent on its physical location. This means that the amount of noise in the scene cannot be known with full precision prior to reconstructing the reflectivity profile of scene. To obtain an estimate of the phase noise power, we assume that the scatterers are located near the middle of the scene. This removes the explicit dependence on their location $\tau_{i}$ while giving a reasonable estimate for the noise. Equation 6 can therefore be simplified to

$$
\left\langle\left\|\mathbf{n}_{\text {phase }}\right\|_{2}^{2}\right\rangle \propto \sum_{i} R_{i} \Longrightarrow\left\langle\left\|\mathbf{n}_{\text {phase }}\right\|_{2}^{2}\right\rangle \propto\left\|\mathbf{x}_{\text {noiseless }}\right\|_{2}^{2} .
$$

The energy in the noise can be estimated as a scaled version of the measured noiseless signal power. Uncertainty in the optical frequency output from the laser, described in Section 4, will also manifest itself in an indistinguishable 
fashion limiting the usefulness of theoretical estimates for the phase noise. Since phase noise is the dominant source of noise, we ignore the contribution of shot noise in our CS reconstructions.

Using the restricted isometry property, ${ }^{16}\left\|\mathbf{x}_{\text {noiseless }}\right\|_{2}^{2} \approx\left\|\mathbf{y}_{\text {noiseless }}\right\|_{2}^{2},\|\mathbf{n}\|_{2}^{2}$ can be estimated from the noisy measurements $\mathbf{y}$ using:

$$
\|\mathbf{y}\|_{2}^{2}=\left\|\mathbf{y}_{\text {noiseless }}\right\|_{2}^{2}+\left\langle\|\mathbf{z}\|_{2}^{2}\right\rangle=\left\|\mathbf{x}_{\text {noiseless }}\right\|_{2}^{2}+\left\langle\|\mathbf{n}\|_{2}^{2}\right\rangle
$$

solving for $\left\langle\|\mathbf{n}\|_{2}^{2}\right\rangle$ and letting $\left\|\mathbf{x}_{\text {noiseless }}\right\|_{2}^{2}=\operatorname{SNR}\left\langle\|\mathbf{n}\|_{2}^{2}\right\rangle$ we obtain:

$$
\left\langle\|\mathbf{n}\|_{\mathbf{2}}^{\mathbf{2}}\right\rangle=\frac{1}{1+\mathrm{SNR}}\|\mathbf{y}\|_{2}^{2} .
$$

This estimate of the noise is subsequently used in the CS reconstruction of the reflectivity profile of the scene described in the next section. To estimate the SNR in our particular implementation due to phase noise, the CS reconstruction of a single scatterer reflector placed at $\approx 7 \mathrm{~mm}$ of path length mismatch was performed. The SNR was increased until false scatterers start to appear. It is found that in our particular experiments, the total signal to noise ratio $\left(\mathrm{SNR}=\|\mathbf{x}\|_{2}^{2} /\left\langle\|\mathbf{n}\|_{2}^{2}\right\rangle\right.$ ) is equal to $10 \mathrm{~dB}$.

\section{RECONSTRUCTION RESULTS}

To demonstrate the imaging capability of the compressed sensing OCT setup at all depths, a thin microscope slide, approximately $1.5 \mathrm{~mm}$ thick, is used to create a scene with two scatterers. 1000 optical frequencies, nominally spaced $5 \mathrm{GHz}$ apart, are addressed, at repetition rate of $1 \mathrm{kHz}$, in a predetermined randomized order using 3 arbitrary waveform generators as shown in Figure 1. One hundred consecutive samples $(m=100)$ are used to reconstruct the scene. The reconstruction is then achieved by solving the $l_{1}$-analysis problem: ${ }^{11}$

$$
\begin{array}{cl}
\operatorname{minimize} & \|\mathbf{W} \mathbf{x}\|_{1} \\
\text { subject to } & \|\mathbf{A x}-\mathbf{y}\|_{2}<\epsilon=\left\langle\|n\|_{2}^{2}\right\rangle^{1 / 2},
\end{array}
$$

where $\mathbf{x}$ is a vector with the $N=1000$ components corresponding to the real and imaginary components of the reflectivity at the $N / 2$ resolution limited locations in the scene, $\mathbf{A}$ is the corrected sampled Fourier matrix corresponding to the addressed optical frequencies from the laser, $\mathbf{y}$ contains the measurements. $\mathbf{W}$ is a matrix of size $4000 \times 1000$, that interpolates the vector $\mathbf{x}$ in between the Fourier limited locations using a Hamming window. W helps promote sparsity for targets that do not lie exactly on the discretized grid. To improve the reconstruction, reweighing ${ }^{14}$ of the $\mathbf{W x}$ coefficients was done 4 times. We reconstruct multiple scenes with two reflectors, formed by the two facets of an acrylic slab, with the slab placed at different levels of path length mismatch in the scene. The results of the reconstruction are illustrated in Figure 4(a) where each instance of the scene is shown in a different line style. The reconstructions show good agreement of the reflector strengths between scans. It is important to note that the number of weak false targets that appear in the realization of the scene where the glass slide is located further from the mirror increases slightly. This is attributed to the fact that $\epsilon$ was tuned for a target at a path length mismatch of $7 \mathrm{~mm}$. Targets further away contain more noise which manifests itself as false weak targets.

To demonstrate the capability of reconstructing targets with different relative strengths, located in vastly different locations, we add a second microscope slide, $\approx 0.3 \mathrm{~mm}$ thick, $10 \mathrm{~mm}$ away from the first. The same $l_{1}$ minimization problem is solved and the results are shown in Figure 4(b). Once again, the relative strengths and locations of all four targets are correctly identified.

One detail worth consideration when using compressed sensing is the speed at which one can recover the original signal. In the current Matlab TFOCS ${ }^{17,18}$ implementation, one reconstruction, including the 4 reweighing steps takes approximately 1 minute on a desktop computer. For a full 3D scan, containing $100 \times 100$ lateral locations, the reconstruction of the full scene would take approximately 1 week. Parallelization can help reduce the overall reconstruction time at the expense of added programming complexity. We expect that this reconstruction time can be significantly improved; however, the usefulness of a CS acquisition scheme may be most important in to cases where acquisition time, or optical energy is a premium. This may eventually prove to be useful in dynamic biological processes where the dose of optical power is limited so as not to damage the specimen. 

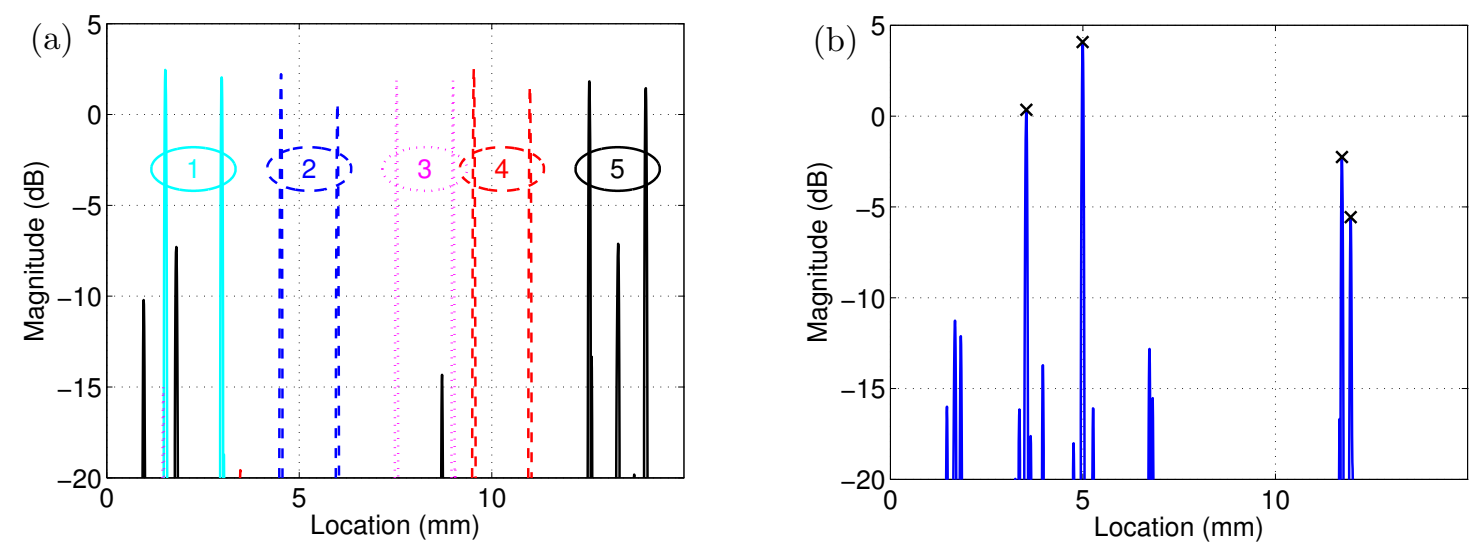

Figure 4: (a) CS reconstruction of a two target scene at various levels of path length mismatch with $m=$ $100, N=1000$. The motorized staged is moved between different instances of the scene to change the path length mismatch. Each instance is depicted with a different line style. The scene is composed of a single acrylic slab, 1.5 mm thick. (b) CS reconstruction of a scene with two acrylic slabs (4 reflectors) of the reflectors slightly misaligned to change their relative strength. The strength and location of all 4 reflectors, marked by the symbol $\times$, are correctly recovered.

\section{CONCLUSIONS}

To conclude, a demonstration of a swept source compressed sensing optical coherence tomography is shown to decrease the number measurements taken to reconstruct a sparse scene, thereby reducing the acquisition time. A compression ratio of 10 for a two scatterer scene and a compression ratio of 5 for a scene with four scatterers of different relative strengths were achieved. While the current scan acquisition time is not yet comparable to that of the state of the art OCT setups, better dynamic characterization of the output frequency of the Vernier tunable laser promises to make this a competing technology. The usefulness of this technique will ultimately depend of the reconstruction speed of the depth profile from the compressed samples.

\section{ACKNOWLEDGMENTS}

The authors would like to thank John J. Bruer and Roarke Horstmeyer for their insightful discussions. This work was supported in part by the Defense Advanced Research Projects Agency (DARPA) Knowledge Enhanced Compressive Measurements program (KECoM).

\section{REFERENCES}

[1] Drexler, W. and Fujimoto, J. G., eds., [Optical Coherence Tomography Technology and Applications], Springer Berlin Heidelberg, (2008).

[2] Jayaraman, V., Cole, G. D., Robertson, M., Burgner, C., John, D., Uddin, A., and Cable, A., "Rapidly swept, ultra-widely-tunable $1060 \mathrm{~nm}$ MEMS-VCSELs," Electronics Letters 48, 1331-1333 (2012).

[3] Coldren, L. A., "Monolithic tunable diode lasers," IEEE Journal of Selected Topics in Quantum Electronics 6, $988-999$ (2000).

[4] Hulme, J. C., Doylend, J. K., and Bowers, J. E., "Widely tunable Vernier ring laser on hybrid silicon," Opt. Express 21, 19718-19722 (2013).

[5] Laroy, R., Morthier, G., Mullane, T., Todd, M., and Baets, R., "Stabilisation and control of widely tunable MG-Y lasers with integrated photodetectors," IET Optoelectronics 1, 35-38 (2007).

[6] Candès, E. J., Romberg, J., and Tao, T., "Robust uncertainty principles: exact signal reconstruction from highly incomplete frequency information," IEEE Transactions on Information Theory 52, 489-509 (2006). 
[7] Engelstaedter, J. P., Roycroft, B., Peters, F. H., and Corbett, B., "Dynamic SMSR measurement of fast SG-DBR laser wavelength switching," Conference on Lasers and Electro-Optics/International Quantum Electronics Conference, CTuQ5 (2009).

[8] Huber, R., Wojtkowski, M., and Fujimoto, J. G., "Fourier Domain Mode Locking (FDML): A new laser operating regime and applications for optical coherence tomography," Opt. Express 14, 3225-3237 (2006).

[9] Vasilyev, A., Satyan, N., Xu, S., Rakuljic, G., and Yariv, A., "Multiple source frequency-modulated continuous-wave optical reflectometry: theory and experiment," Appl. Opt. 49, 1932-1937 (2010).

[10] Needell, D. and Tropp, J. A., "CoSaMP: Iterative signal recovery from incomplete and inaccurate samples," Applied and Computational Harmonic Analysis 26(3), 301-321 (2009).

[11] Elad, M., Milanfar, P., and Rubinstein, R., "Analysis versus synthesis in signal priors," Inverse Problems 23(3), 947 (2007).

[12] Minneman, M. P., Ensher, J., Crawforda, M., and Derickson, D., "All-semiconductor high-speed akinetic swept-source for OCT," Optical Sensors and Biophotonics, 831116 (2011).

[13] Wesstrom, J.-O., Hammerfeldt, S., Buus, J., Siljan, R., Laroy, R., and de Vries, H., "Design of a widely tunable modulated grating Y-branch laser using the additive Vernier effect for improved super-mode selection," in IEEE 18th International Semiconductor Laser Conference, 99-100 (2002).

[14] Candès, E. J., Eldar, Y. C., Needell, D., and Randall, P., "Compressed sensing with coherent and redundant dictionaries," Applied and Computational Harmonic Analysis 31(1), 59-73 (2011).

[15] Venkatesh, S. and Sorin, W. V., "Phase noise considerations in coherent optical FMCW reflectometry," Journal of Lightwave Technology 11, 1694-1700 (1993).

[16] Davenport, M. A., Laska, J. N., Treichler, J., and Baraniuk, R. G., "The pros and cons of compressive sensing for wideband signal acquisition: Noise folding versus dynamic range," IEEE Transactions on Signal Processing 60, 4628-4642 (2012).

[17] Becker, S. R., Candès, E. J., and Grant, M. C., "Templates for convex cone problems with applications to sparse signal recovery," Mathematical Programming Computation 3(3), 165-218 (2011).

[18] Grant, M. and Boyd, S., "TFOCS: Templates for first-order conic solvers." https://cvxr.com/tfocs/, (2013). 